\title{
Flare-Induced Interaction Lengths in Supersonic, Turbulent Boundary Layers
}

\author{
A. Roshko* and G. J. Thomke $\dagger$ \\ Douglas Aircraft Company, El Segundo, Calif.
}

\begin{abstract}
Experimental results are presented for the effects of Mach number, Reynolds number, and corner angle on flare-induced separation of a supersonic, turbulent boundary layer. Measurements were obtained for upstream interaction distance $\ell_{0}$ from the flare to the beginning of the interaction for Mach numbers $2 \leq M \leq 4.5$, boundary-layer thickness Reynolds numbers $10^{5}<R_{\delta}<10^{6}$, and adiabatic wall conditions. Flares of angle $9^{\circ} \leq \alpha \leq 40^{\circ}$ were attached to a hollow-cylinder model of 12 in. diam at either $x_{c}=14$ or 28 in. downstream from the sharp leading edge. It was found that $\ell_{0} / \delta_{0}$ decreases with increasing Mach number and Reynolds number and increases with flare angle. For constant $\alpha$, when $\ell_{0} / \delta_{0}$ is plotted vs the local skin-friction coefficient, $C_{f 0}$, the Mach number dependence disappears. From this observation, a simple correlation formula was obtained and used to compare results from other investigations, and also to correlate incipient separation data.
\end{abstract}

\section{Nomenclature}

$C_{f} \quad=2 \tau_{w} / \rho_{e} U_{e}^{2}$, local skin-friction coefficient

$\ell=$ interaction length, measured upstream from corner

$M \quad$ = Mach number

$P \quad=$ pressure

$r \quad=$ radius of cylinder outer surface

$R_{\Delta} \quad=\rho_{e} U_{e} \Delta / \mu_{e}$, Reynolds number based on

$\Delta=\delta, \delta^{*}, \theta$, or $c=x_{c}$

$T \quad=$ temperature

$U \quad=$ velocity

$x=$ distance downstream from leading edge

of cylinder

$=$ radial distance from cylinder surface

$=$ flare angle, deg

$=$ boundary-layer thickness

= boundary-layer displacement thickness

$=$ boundary-layer momentum thickness

= wetted distance from compression corner, positive upstream

$=$ density

= upstream influence coefficient

\section{Subscripts}

$=$ undisturbed conditions at compression corner

$=$ freestream conditions

$=$ conditions for incipient separation

$=$ undisturbed conditions at beginning of interaction

$=$ plateau

$=$ recovery conditions

$=$ conditions at separation point

$=$ freestream total conditions

$=$ conditions at the surface

\section{Introduction}

$\mathbf{T}$ HE investigations reported in this paper are a natural development from the earlier work of the

Presented as Paper 75-6 at the AIAA 13th Aerospace Sciences Meeting, Pasadena, Calif., Jan. 20-22, 1975; submitted April 1, 1975; revision received March $17,1976$.

Index categories: Boundary Layers and Convective Heat Transfer-Turbulent; Jets, Wakes and Viscid-Inviscid Flow-Interactions; Supersonic and Hypersonic Flow

"Consultant; also Professor of Aeronautics, California Institute of Technology. Fellow AlAA.

†Senior Engineer/Scientist, Aerophysics Laboratory. Member AIAA. authors ${ }^{1}$ on incipient separation at very high Reynolds number which showed trends quite different from those which could be extrapolated from prior results at lower values of Reynolds number. Specifically, in the range $10^{8} \leq R_{c} \leq 10^{9}$, the ramp angle needed to induce separation $\alpha_{i}$ increased with $R_{\mathrm{c}}$. This is opposite to what had been observed at values of $R_{c}$ below $10^{7}$ by Kuehn ${ }^{2}$ and others. However, Settles and Bogdonoff ${ }^{3}$ found that the angle for incipient separation $\alpha_{i}$ does not vary with $R_{c}$, while Law ${ }^{4}$ found that it increases; the latter results tend to confirm the trends of Ref. 1.

It was natural to ask whether the increased "resistance to separation" at high Reynolds number occurs also for values of $\alpha>\alpha_{i}$, i.e., for separated flow. Indeed, the few results obtained ${ }^{\prime}$ indicated that the interaction lengths $\ell_{0}$ and $\ell_{s}$ (which characterize the beginning of the pressure rise and the location of the separation point, respectively, upstream of the ramp), normalized by the boundary-layer thickness $\left(\delta_{0}\right)$ just ahead of the interaction, decreased with increasing $R_{c}$. Other evidence for the decrease of $\ell_{0} / \delta_{0}$ and $\ell_{s} / \delta_{0}$ with increasing $R_{c}$ was also available from the experimental investigations of Refs. 3 and 4 as well as the theoretical results of Refs. 5-7.

It is curious that up to now there has been no cogent account of interaction length behavior in supersonic, turbulent boundary layers. The problem has attracted the attention of many investigators, and various correlations have been attempted, but with little success. A difficulty has been the lack of experimental data for conditions varied systematically over a sufficiently broad range. In a survey of over 100 papers and reports giving experimental results, we found it problematic to sort out trends. The great bulk of the data were in a range of Reynolds number $R_{c}$ from about $10^{5}$ to $10^{7}$, or even narrower - this dictated by the characteristics of the typical small or medium-sized supersonic wind tunnels in which research of this kind was ordinarily conducted. In this range, the flows were transitional more often than not, or had hardly recovered from the effects of transition or tripping, so that the establishment of accurate trends for purely laminar or purely turbulent separation proved difficult. The situation was compounded by the facts that any one investigation was usually conducted over a rather limited range (if any) of Mach number and Reynolds number, and different investigators used different ramp angles, usually with little variation. It was therefore difficult, even with interpolation and extrapolation, to put together a broad picture.

In designing the experiments described here, the aim was to take advantage of the model size and high Reynolds number capability offered by a large wind tunnel so that $\ell_{0} / \delta_{0}$ or $\ell_{0} /$ $x_{c}$ could be varied systematically over as wide a range as 
possible of the relevant parameters, viz., the ramp angle $(\alpha)$, Mach number $\left(M_{c}\right)$, and Reynolds number $\left(R_{c}\right.$ or $\left.R_{\delta}\right)$. It would have been desirable to continue with the experimental arrangement of Ref. 1, where wall boundary-layer thicknesses ranged from 3-6 in., but model blockage and shock-wave induced interference effects would have appreciably diminished the range of $\alpha$ and $\ell_{0} / \delta_{0}$ for which valid data could be obtained. Further study indicated that the desired goals could best be achieved by using a 1-ft-diam hollow cylinder to which flares of various angles could be attached. Experimental conditions would cover the Reynolds number range $10^{6} \leq R_{\delta} \leq$ $10^{7}$-i.e., one decade lower than that of Ref. 1 . With a sharp leading edge, supersonic flow through the inlet, and boundary-layer thicknesses small compared to the cylinder radius $(\delta / r<0.07)$, the boundary layer and adjacent flowfield would be similar to those for a flat plate. However, for large separation regions ahead of the larger flares, an effect of the axisymmetric parameter $x_{c} / r$ could be expected.

\section{Experimental Arrangement and Procedure}

\section{Wind Tunnel}

The experiments were made in the $4 \times 4$ - $\mathrm{ft}$ Trisonic Wind Tunnel (blowdown) located at the McDonnell Douglas Aerophysics Laboratory in El Segundo, Calif. Test-section Mach number was varied from 2.0-4.5; unit Reynolds number was varied from $0.5-2$ million/in. by adjusting total pressure. Depending upon Mach number, total temperatures were in the range from $520-630^{\circ} \mathrm{R}$ and provided nearly adiabatic wall conditions for the model. During a run, total temperature decreased slightly (no more than $10^{\circ}$ over the total run interval, and less than $5^{\circ}$ during a data measurement interval).

\section{Model and Instrumentation}

The basic part of the model, the hollow cylinder, was a steel tube with 11.94 in. $0 . d$. and a wall thickness of 0.34 in. The outer surface of the tube was machined and polished to a $32-\mu$ in. finish. The inlet section was chamfered internally at an angle of $4^{\circ}$ and honed to provide a sharp leading edge. Compression corners were formed on the outer surface of the cylinder by attaching to it two flare halves, separated by splitter plates, as shown in Fig. 1. The feasibility of using splitter plates had been studied in some previous, unpublished work (related to that in Ref. 8) on an axisymmetric, downstreamfacing step, where it was found that the presence of splitter plates in the base region had no effect on the pressure distribution or the extent of the separated region, even when the circumferential distance between the plates was as small as $45^{\circ}$ (compared to $180^{\circ}$ in the present case).

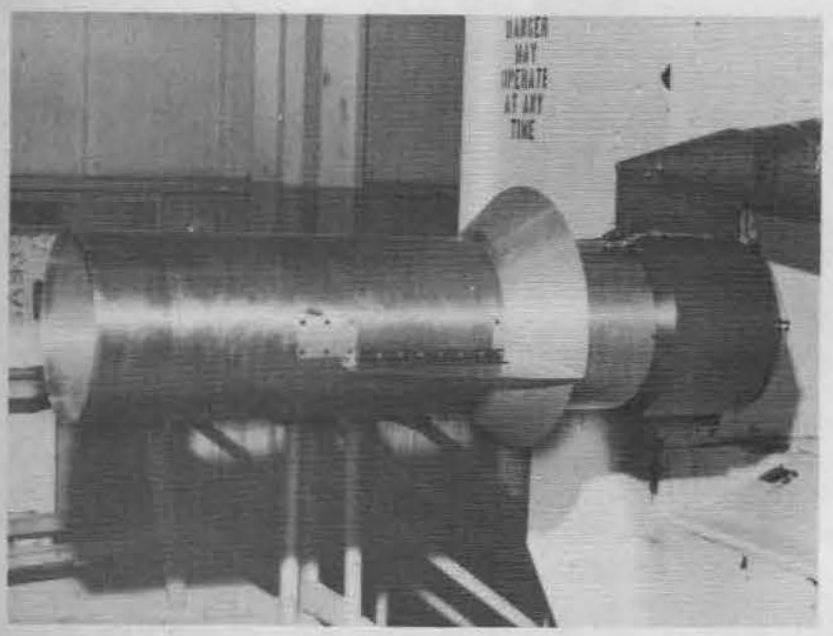

Fig. 1 Model with $40^{\circ} / 30^{\circ}$ half-flares at $x_{c}=28$ in.
Flares were made of aluminum and paired according to the following values for $\alpha$ : 9-13, 19-20, 22-25, 25-25 (with and without splitter plates), $27-35$, and $30-40^{\circ}$. Slant lengths ranged from 4.6 in. $\left(\alpha=9^{\circ}\right)-5.9$ in. $\left(\alpha=40^{\circ}\right)$. Flare pairs could be positioned at two locations on the cylinder, $x_{c}=14$ or 28 in. measured downstream from the leading edge of the cylinder to the leading edge of each flare. Corresponding values of the axisymmetric parameter $x_{c} / r$ are 2.33 and 4.66, respectively.

The cylinder surface was instrumented with 146 orifices $(0.03$-in. diam) for sensing surface pressure, and a thermocouple for sensing surface temperature. Flares of angle $\alpha$ $\leq 25^{\circ}$ were instrumented with 16 orifices. Pressures and temperatures were measured with conventional wind-tunnel instrumentation. Schlieren photographs were taken periodically during each run to assist in evaluation of test results, and a traversing probe was used to obtain boundary-layer pitotpressure profiles.

\section{Procedure}

The flare experiments were run at nominal Mach numbers of $2,2.5,3,3.3,3.5,3.7,4$, and 4.5 , and at tunnel unit Reynolds number of $0.5,1.0,1.5$, and 2.0 million/in. Thus, by varying both $x_{i}$ and unit Reynolds number, an eightfold change in $R_{c}$ was possible. Wall-temperature to recoverytemperature ratios for the model were in the range $0.95 \leq T_{w}$ / $T_{r} \leq 1.05$. Mach number, Reynolds number, and flare angle were held constant during a run.

The model was supported in the center of the tunnel as shown in Fig. 1. Various checks showed that the model was aligned parallel to the tunnel flow. Data repeatability was excellent. Splitter plate effects were evaluated at $M=2.5\left(\ell_{0} /\right.$ $\delta_{0}=4$ ) and $M=3$ by comparing results obtained at $x_{c}=28$ in. for the $\alpha=25-25^{\circ}$ pair with and without plates. For each case, plate effects could not be detected.

Pitot-pressure profiles for the undisturbed boundary layer on the cylinder (without flares) were obtained at $x=14$ and 28 in. for the ranges of $M$ and $R_{c}$ covered in this study. At these locations, representative values of the boundary-layer thickness were $\delta=0.195$ and 0.355 in. Boundary-layer trip devices were not employed. According to data presented by Pate and Schueler ${ }^{y}$, the position of natural transition on the cylinder was estimated to be from $2-8$ in. from the leading edge, depending on $M$ and $R_{c}$.

\section{Results}

The experimental results consisted principally of determinations of the various parameters describing the undisturbed boundary layer on the cylinder, Schlieren photographs of the interaction region, and surface-pressure distributions.

\section{Boundary-Layer Parameters}

Boundary-layer Mach number profiles were computed from the measured pitot-pressure values with wall pressure assumed to be constant through the layer. Velocity and density profiles were calculated assuming the temperature profile to be given by

$$
\begin{aligned}
& T / T_{e}=\left(T_{w} / T_{e}\right)+\left[\left(T_{r} / T_{e}\right)\right. \\
& \left.\left.-\left(T_{w} / T_{e}\right)\right]\left(U / U_{e}\right)-\left[\left(T_{r} / T_{e}\right)-l\right)\right]\left(U / U_{e}\right)^{2}
\end{aligned}
$$

The outer portion of the velocity profile was assumed to be represented by a $1 / n$-type power law, and $n$ was taken to be equal to the slope of the best straight-line fit to logarithmic values of $y$ and $U / U_{e}$ for $y>0.03$ in. Values for $n$ ranged from 5.5-6.5 at $x_{c}=14 \mathrm{in}$. and 6.0-7.5 at $x_{c}=28 \mathrm{in}$. 


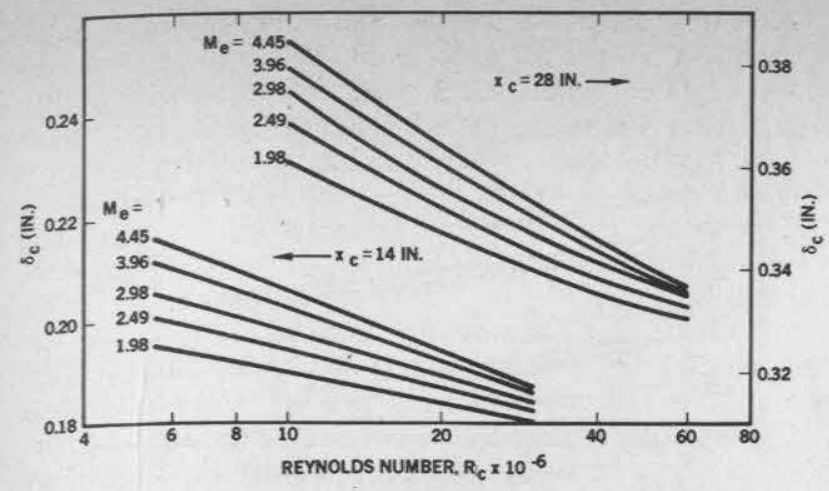

Fig. 2 Boundary-layer thickness at $x_{c}=14$ and 28 in.

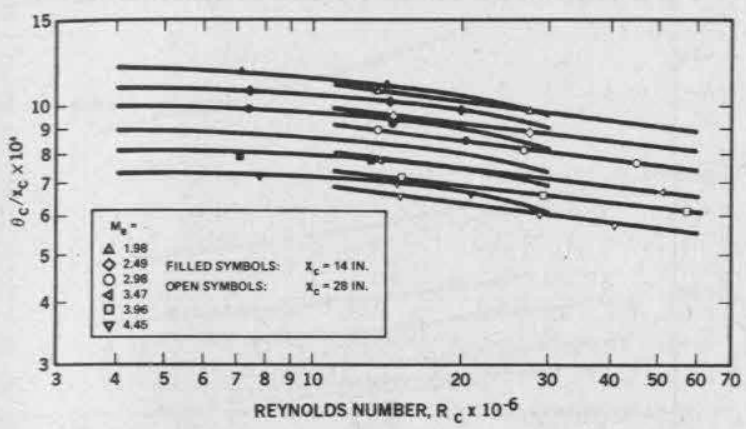

Fig. 3 Momentum-thickness at $x_{c}=14$ and 28 in.

The value of $y$ at $U / U_{e}=0.995$ was chosen for $\delta$. The curves of Fig. 2 represent the smoothed values of $\delta$ obtained for the undisturbed boundary layer at $x=14$ and 28 in. for the range of Mach number and (unit) Reynolds number shown. Data scatter about each curve is less than $3 \%$. These results indicate that dependence of $\delta$ on Mach number is small and decreases with increasing Reynolds number.

The momentum-deficit thickness was determined by graphically integrating the expression

$$
\theta=\int_{0}^{\infty}\left(\rho U / \rho_{e} U_{e}\right)\left[1-\left(U / U_{e}\right)\right] \mathrm{d} y
$$

Values obtained for $\theta_{\text {s }}$ are plotted in Fig. 3. To scale the thickness parameters to values of $x$ other than $x=14$ and 28 in., use was made of the following equations: $\dagger$

$$
\begin{gathered}
\delta / x=0.1215 R_{x}^{-1 / 8} \\
\theta / x=0.0125 e^{-M / 5} R_{x}^{-1 / 8}
\end{gathered}
$$

Unless otherwise noted, all values for the local skin-friction coefficient $C_{f}$ used in this paper were obtained from the equations of Hopkins ${ }^{10}$ based on the method (II) of Van Driest, into which were put measured or estimated values for $M_{c}, T_{r}$, and $R_{\theta}$ plus the adiabatic wall condition, $T_{n} / T_{r}=1$. Sutherland's formula for viscosity was also used.

\section{Pressure Distributions}

An example of a measured pressure distribution is shown in Fig. 4 for the case $\alpha=25^{\circ}$. The method of defining the upstream interaction length $\ell_{0}$ illustrated in the figure, is the one used by Settles and Bogdonoff. ${ }^{3}$

†These are empirical fits that we have made to a large body of experimental results obtained by us for an adiabatic, flat-plate boundary layer at $10^{5} \leq R_{\delta} \leq 10^{7}$ and $1.8 \leq M \leq 5$.

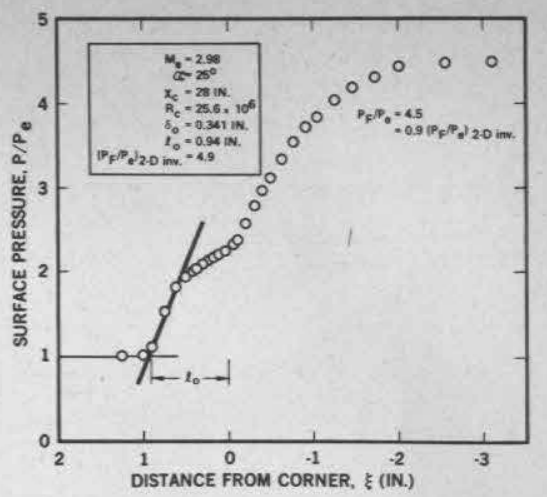

Fig. 4 Definition of interaction length.

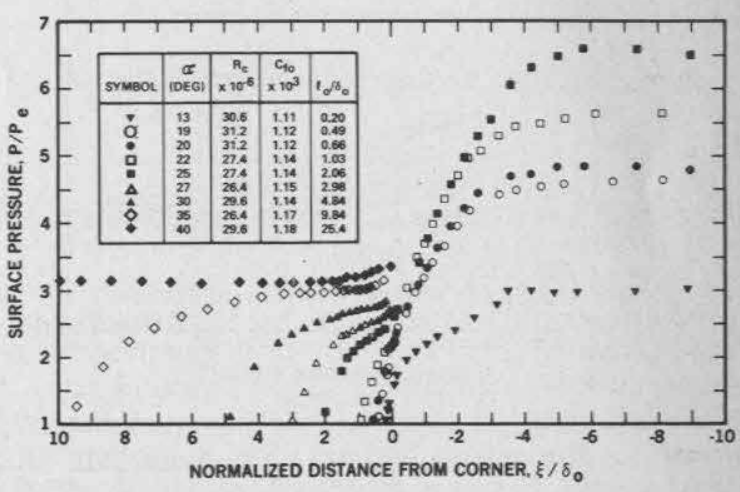

Fig. 5 Surface pressure distributions, $M_{e}=3.96, x_{c}=28$ in.

Figure 5 presents a set of pressure distributions obtained at $M_{e}=3.96$ for a nominal Reynolds number $R_{c}=30 \times 10^{6}$. (Flares of angle $\alpha>25^{\circ}$ were not provided with pressure taps). The listed values of $C_{f 0}$ are computed for the point at the beginning of interaction, $x_{0}=x_{c}-\ell_{0}$, and distances from the corner $\xi$ are normalized with $\delta_{0}$, the value for the undisturbed boundary-layer thickness at $x_{0}$. For $\alpha=40^{\circ}$ the interaction begins at $\xi / \delta_{0}=25.4$, so only the portion of the pressure distribution near the corner has been plotted in Fig. 5. The nearly constant pressure in this portion is taken to be the plateau pressure, $P_{P}=3.12 P_{e}$. Plateau pressure values obtained at the Mach numbers of this experiment are close to those given for upstream-facing steps by the correlations of Zukoski ${ }^{11}$ and of Werle ${ }^{12}$; they were found to be insensitive to Reynolds number, as Zukoski had found earlier.

\section{Interaction Length}

The complete set of values of the upstream interaction length $\ell_{0}$ obtained over the range of all the parameters is presented in Figs. 6 and 7 , in which the interaction lengths have been normalized by $x_{c}$ and plotted against $R_{c}$. While the trends shown in these basic data plots are interesting, a more significant plot would be based on a boundary-layer thickness parameter instead of $x_{c}$. When values of $\ell_{0} / \Delta$ (where $\Delta=\delta, \delta^{*}$, or $\theta$ ) are plotted against $R_{\Delta}$, it is found that $\ell_{0} / \Delta$ decreases with increasing $R_{\Delta}$ for the whole range of flow parameters, whether $\Delta$ be evaluated at $x_{0}$ or $x_{c}$; this is similar to the trends found by Settles and Bogdonoff ${ }^{3}$ and by Law ${ }^{4}$ at $M=3$ for $\Delta$ $=\delta$. However, these plots are not shown here because a better way to present the data was found, one in which the dependence on Mach number disappears.

For reasons which are not yet understood, when $\ell_{0} / \delta_{0}$ is plotted against $C_{f 0}$, the data for different values of Mach number (excluding the $M_{e}=1.98$ data) fall onto a single curve for each value of $\alpha$, as may be seen in Fig. 8. Values of $C_{f 0}$ were determined as previously explained. The data from the present experiments are in the range $10^{5}<R_{\delta}<10^{6}$, but in- 

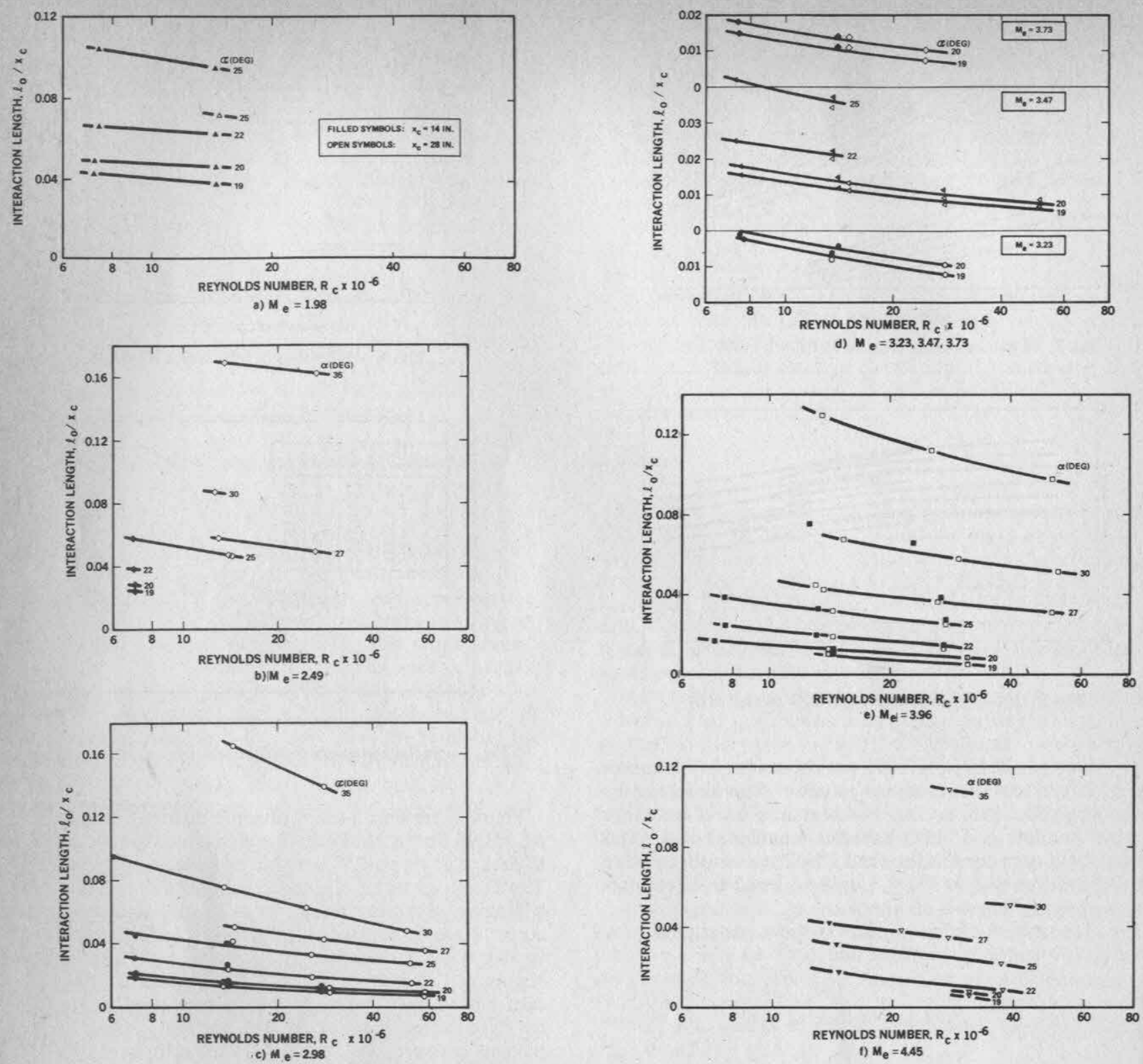

Fig. 6 Effect of flare angle and Reynolds number on interaction length: a) $M_{e}=1.98$; b) $M_{e}=2.49$; c) $M_{e}=2.98$; d) $M_{e}=3.23,3.47,3.73 ;$ e) $M_{e}=3.96 ;$ f) $M_{e}=4.45$.

cluded on the plots are data in the next higher decade from our previous study, ${ }^{1,13}$ which also fall onto the correlation. It is remarkable that the data for each value of $\alpha<35^{\circ}$ (and excluding those for $M_{e}=1.98$ ) fall on a straight line. We denote the slope of the line by $\sigma$ and the intercept skin-friction value by $C_{f 0}^{*}$. For $\alpha=35^{\circ}$, some liberty has been taken in fitting a straight line to part of the correlation [see remarks following Eq. (5), below]. A discussion of factors contributing to the nonconforming behavior exhibited by the data obtained for $M_{e}=1.98$ and for $\alpha>35^{\circ}$ is given below. Data for $\alpha=9^{\circ}$ and $13^{\circ}$ are not included in Figs. 6 and 8 because only a few data points were obtained, and also because of the difficulty (due to orifice spacing) in obtaining precise values of $\ell_{0}$ from surface-pressure measurements for small $\alpha$. However, four data points obtained for $\alpha=13^{\circ}, x_{c}=28$ in., and $M_{e}=2.98$ and 3.96, when plotted in the format of Fig. 8, were in agreement with a straight line passing through $C_{f 0}^{*}=0.0008$ with slope $\sigma$ $=400$.

The straight-line fits may be represented by the equation

$$
\ell_{0} / \delta_{0}=\sigma\left(C_{f 0}-C_{f 0}^{*}\right)
$$

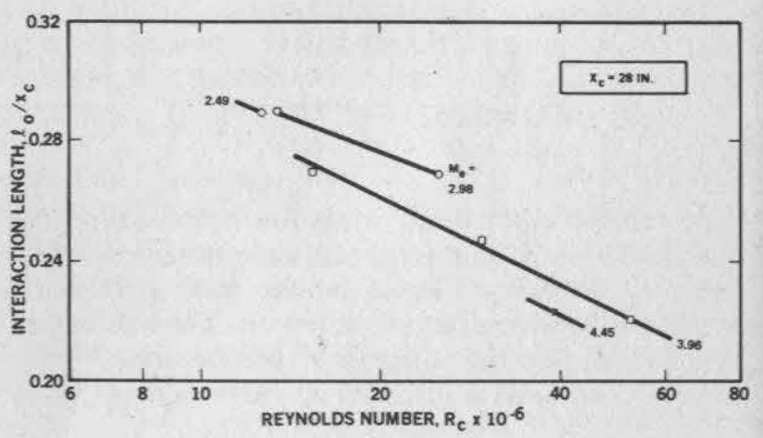

Fig. 7 Interaction length for $\alpha=40^{\circ}$.

in which, in addition to the intercept parameter $C_{f 0}^{*}(\alpha)$, there appears the upstream-influence coefficient $\sigma$, i.e., the slope of the line, which also depends on $\alpha$. Values of $\sigma(\alpha)$ are well fitted by the empirical equation

$$
\sigma=10^{3}(\alpha / 18.29)^{2.81}
$$



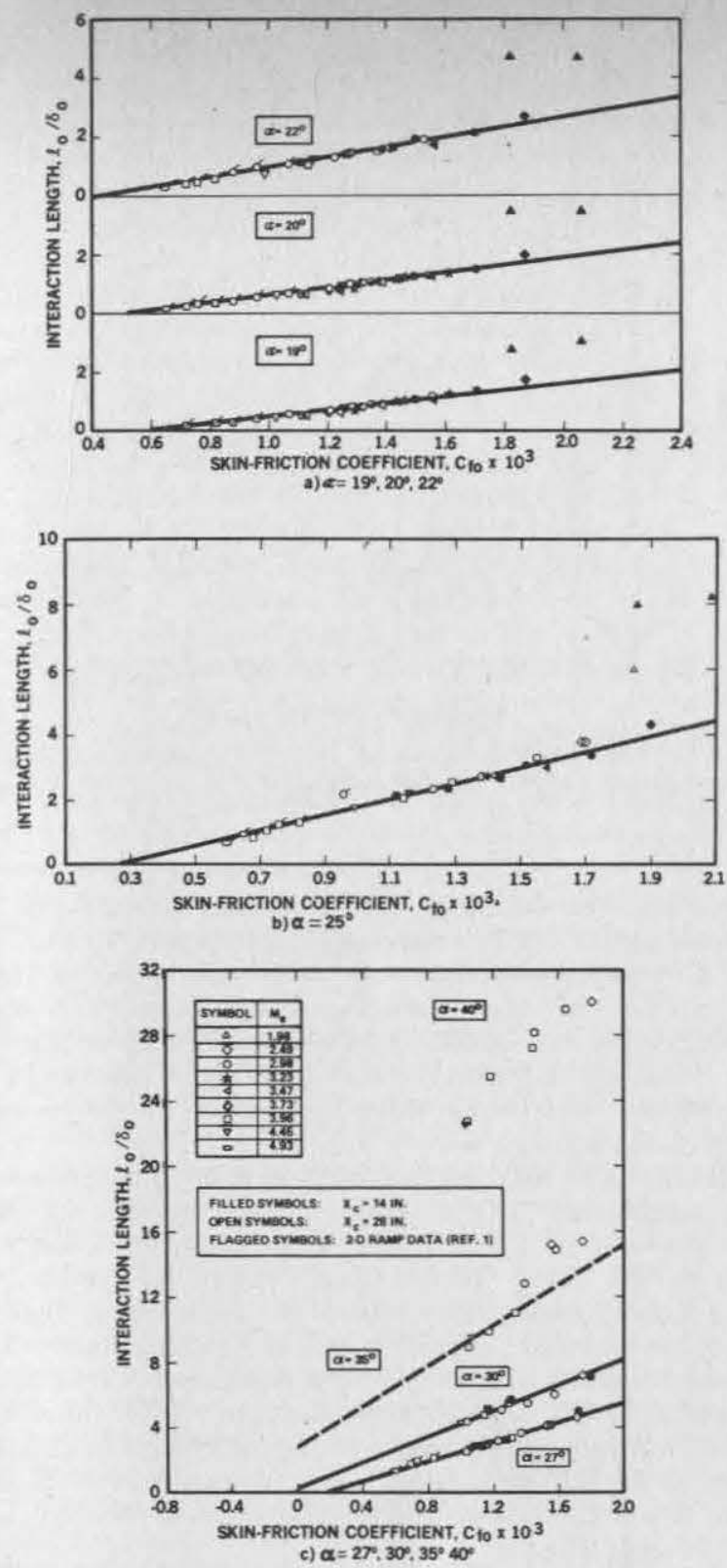

Fig. 8 Correlation of interaction length with local skin-friction coefficient: a) $\alpha=19^{\circ}, 20^{\circ}, 22^{\circ}$; b) $\alpha=25^{\circ}$; c) $\alpha=27^{\circ}, 30^{\circ}, 35^{\circ}, 35^{\circ}, 40^{\circ}$.

(which was used a posteriori to fit part of the data for $\alpha=35^{\circ}$ in Fig. 8). Values of $C_{j 0}^{*}(\alpha)$ are well fitted by the equation

$$
C_{f 0}^{*}=10^{-3}\left(1-0.001189 \alpha^{2}\right)
$$

It is not clear what physical interpretation, if any, to attach to the above curve-fitting equations. The literal implication of Eq. (4) is that, for each $\alpha$, the upstream interaction length $\left(\ell_{0}\right)$ becomes zero at a sufficiently high Reynolds number $R_{\dot{S}}^{*}$ corresponding to $C_{j 0}^{*}$ (the separation length $\ell_{s}$ would become zero at somewhat lower Reynolds number). Of course it is not physically realistic for upstream influence to become precisely zero at finite Reynolds number, because a sublayer next to the wall will always be subsonic. A further interesting result is that, according to Eq. (6), $C_{j 0}^{*}$ becomes zero for $\alpha=29^{\circ}$, implying that for $\alpha>29^{\circ}$ there is always an upstream influence (and for somewhat larger $\alpha$ there is always separation) no matter how high the Reynolds number.

Whatever the physical significance of the parameters $C_{j 0}^{*}$ and $\sigma$ defined in this way, Eq. (4) can be used an an interpolation formula for the interaction length $\left(\ell_{0}\right)$ as a function of $\alpha$ and $C_{f 0}$. Figure 8 shows one format for displaying

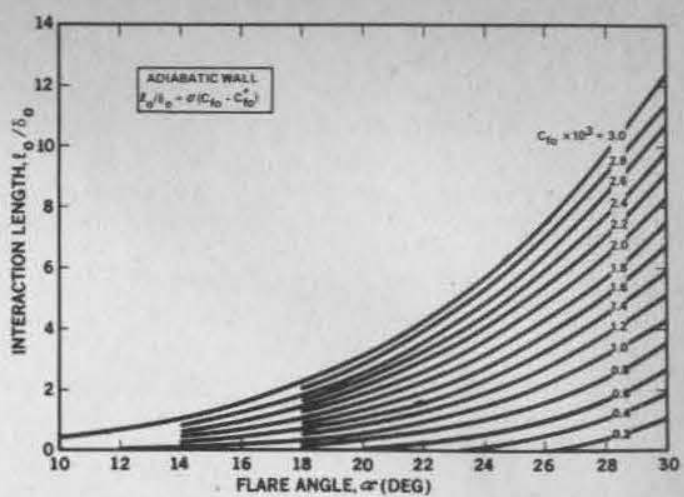

Fig. 9 Upstream influence due to a flare.

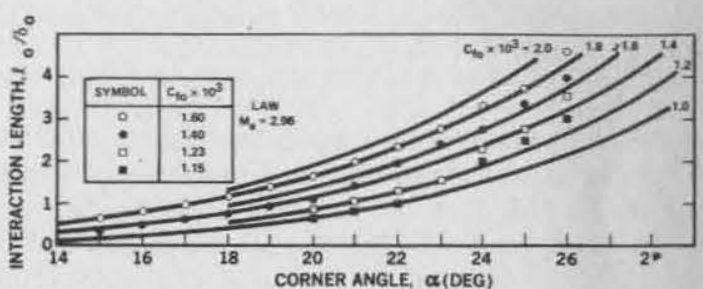

Fig. 10 Comparison between present correlation and experimental results of $\mathrm{Law}^{4}$.

this dependency; another is given in Fig. 9 for flare angles up to $30^{\circ}$ and values of $C_{f 0} \leq 0.003$. Higher values of $C_{f 0}$ would be in the transition range of Reynolds number for the applicable range of Mach numbers $2.5 \leq M_{e} \leq 5$. In fact, the correlation should be used with caution for $C_{j 0} \geq 0.002$, which was the largest value in the present experiments. Over the range of data from which Eq. (4) was derived, there is no effect of the axisymmetric parameter $x_{c} / r$ (cf. Fig. 8 for $x_{\mathrm{c}}=14$ and 28 in.) and therefore the correlation is applicable to twodimensional flow over a ramp.

Law's ${ }^{4}$ experimental results on a two-dimensional (flatplate/ramp) configuration for $M_{e}=3$ and $10^{5}<R_{\delta}<10^{6}$, conditions comparable to that of the present study, are for a broad range of $\alpha$ at 4 discrete values of $R_{\delta}$. His data are therefore compared in Fig. 10 with the correlation in the form of Fig. 9. The values of $C_{f 0}$ were calculated by us as described previously, with the assumption $T_{w} / T_{r}=1$, from data provided by Law. ${ }^{14}$ The excellent agreement with part of Law's data and disagreement (up to $20 \%$ ) with other parts is difficult to reconcile. Apart from quantitive differences, there are curiously abrupt changes (compared to the smooth correlation curves) at about $\alpha=22^{\circ}$. This comparison between our data (represented by the correlation) and Law's is not entirely satisfactory.

The recent data of Settles et al. ${ }^{15}$ cover a somewhat smaller range of $\alpha$ than Law's but a larger number (22) of $R_{\delta}$ values. It is therefore more practical to compare the data with the correlation in the form of Fig. 8. This is shown in Fig. 11. All data are for $M_{e} \simeq 3$ and slightly heated wall conditions (we estimate $T_{w} / T_{r}=1.2$ from conditions cited in Ref. 15). A few data were obtained at $10^{5}<R_{\delta}<10^{6}$ with an axisymmetric (ogive-cylinder/flare) model for which the axisymmetric parameter was $x_{c} / r=14$. The majority of the data were obtained with a two-dimensional (flat-plate/ramp) configuration in a range of Reynolds number, $10^{6}<R_{b}<10^{7}$, comparable to that of Ref. 1 .

Note that in Fig. 11 the experimental data are in terms of conditions at the corner $\delta_{c}$ and $C_{f c}$, whereas the correlation curves are in terms of $\delta_{0}$ and $C_{f 0}$. Since the data extend only over the range $0<\ell_{0} / \delta_{\mathrm{c}}<1.5$, the differences are negligibly small. The notation $\ell_{0} / \delta_{c}$ and $C_{f c}$ used in Fig. 11 correspond, respectively, to the notation $\Delta x / \delta_{0}$ and $C_{f 0}$ used in Figs. 15 and 16 and Tables 1 and 2 of Ref. 15 . Agreement between the 


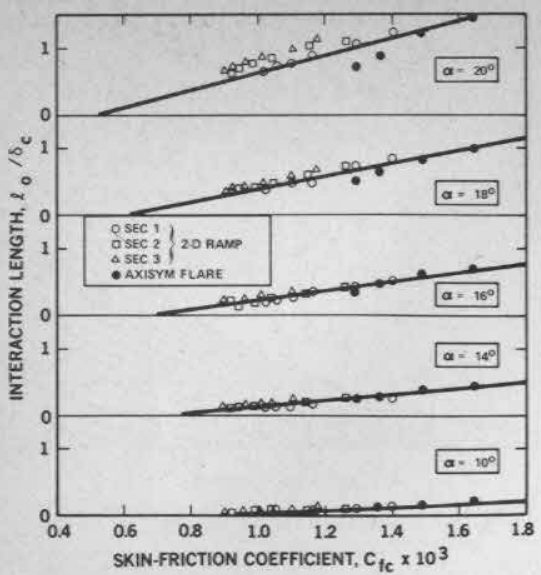

Fig. 11 Comparison between present correlation and experimental results of Settles et al. ${ }^{15}$ at $M_{e}=2.90$.

correlation and experiment is good for $10^{\circ} \leq \alpha \leq 18^{\circ}$. For $\alpha$ $=20^{\circ}$ the agreement is reasonable, considering the variation in the experimental data; the values measured at Sec. 2 and 3 for $\alpha=20^{\circ}$ are consistently higher than those obtained at Sec. 1 by about $\Delta\left(\ell_{0} / \delta_{c}\right)=0.15$. We conclude that the experimental data of Ref. 15 tend to confirm our correlation.

\section{Incipient Separation}

Incipient separation continues to be a vexing problem. Since its earliest study, ${ }^{2}$ subsequent investigations ${ }^{1,3,4,15-18}$ have continued to produce disagreements. This can be attributed largely to the use of different experimental criteria for defining incipient separation conditions. An excellent review of the situation has been given by Appels ${ }^{18}$ who also carried out an investigation of his own at $M=3.5$ over the range $9 \times 10^{4}<R_{\delta}<7 \times 10^{5}$ and at $M=5.4$ over the range $2 \times 10^{5}<R_{\delta}<6 \times 10^{5}$. Appels points out that flow reversal occurs first in the sublayer at small values of $\alpha$; the result is a "small separation" region which can be detected, for example by surface-flow techniques, and which grows quite slowly with increasing $\alpha$, as Spaid and Frishett ${ }^{16}$ had noted earlier. At a sufficiently large value of $\alpha$ the outer portions of the boundary layer become involved and the separation length increases with increasing $\alpha$ at a rate that is several times greater than the growth rate observed for the small separation region. These observations led Appels to define two values for $\alpha_{i}$ at each of his $R_{\delta}$ conditions: the first (low) value of $\alpha_{i}$ corresponds to the onset of the small separation region, the second (high) value of $\alpha_{i}$ characterizes the onset of the high growth rate or "large separation" region.

In the present investigation we did not measure $\alpha_{i}$. Some rough determinations were made from shock geometry on Schlieren pictures, but we did not consider these to be sufficiently accurate for inclusion here. However, the correlation [Eq. (4)] that has been obtained for upstream influence length can be used for exploring the behavior of $\alpha_{i}$. This follows from the observation that the separation point, when it occurs, is always downstream of the beginning of interaction, $\ell_{s}<\ell_{0}$. Thus, each straight line in Fig. 8 passes through a value of $\ell_{0} / \delta_{0}>0$ that corresponds to incipient separation $\left(\ell_{5}=0\right)$. This value of $\left(\ell_{0} / \delta_{0}\right)_{i}$ for incipient separation may depend on the parameters of the problem, but an examination of pressure distributions from the present work and Ref. 1 suggests that it may be relatively constant.

To examine this we have plotted the correlation [Eq. (4)] in Fig. 12 with $\ell_{0} / \delta_{0}$ as a parameter - this is the third way of presenting the correlation. Also plotted on this figure are experimental values of $\alpha_{i}$ from various sources ${ }^{1,4,15-18}$ who used either the surface flow ("liquid") technique, the first appearance of a separation shock at the outer edge of the shear layer, or the orifice-dam ${ }^{1,8,13}$ technique to make their deter-

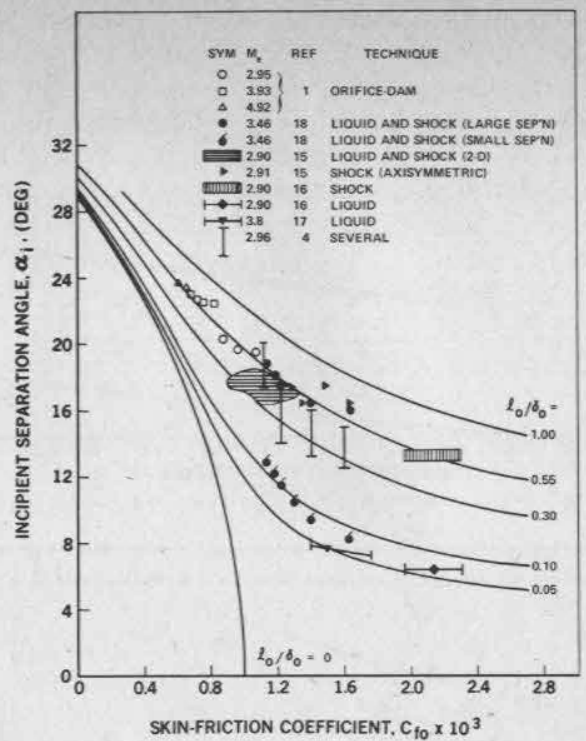

Fig. 12 Experimental values of incipient separation conditions superimposed on the present correlation for upstream influence length.

minations. It is seen that Appels' data from the "small-separation" criterion correspond to $\left(\ell_{0} / \delta_{0}\right)_{i}$ $\doteq 0.1$, while those from the "large-separation" criterion correspond to $\left(\ell_{0} / \delta_{0}\right)_{i} \doteq 0.55$. Other data, ${ }^{16,17}$ which correspond to the "small" criterion are in fair agreement with the value $\left(\ell_{0} / \delta_{0}\right)_{i}=0.1$, while those $e^{1,4,15,16}$ presumed to correspond with the "large" criterion lie in a band $0.3<\left(\ell_{0} / \delta_{0}\right)_{i}<0.7$

In Fig. 12 we have not included values of $\alpha_{i}$ determined by the appearance of a kink in the surface pressure distribution. ${ }^{2,15,16}$ This is the least sensitive indicator of separation onset; furthermore, there is considerable disagreement among the results of the different investigators using the technique, especially at low Reynolds number; the results from Ref. 16 for incipient separation correspond to values of $\left(\ell_{0} / \delta_{0}\right)_{i}$ slightly larger than unity while those from Ref. 2 at similar (low) values of $R_{\delta}$ correspond to values as large as 3 . The implication is that boundary-layer profiles were different, perhaps due to differences in recovery from transition or tripping.

This method of presenting the incipient separation data has the effect of compressing a large range of Reynolds number into a small corresponding range of $C_{f}$. The effect is dependent upon Mach number and becomes most pronounced as $C_{f} \rightarrow 0$. With this compression, it is possible to better see the relation of data over a large range of Reynolds number and the effects of data scatter. Viewing the results collectively, we see an independence of $M$ (as in our basic correlation) and a trend for increasing $\alpha_{i}$ with decreasing $C_{f 0}$ (increasing $R_{\Delta}$ ). An interesting observation is that the limiting value predicted for $\alpha_{i}\left(\right.$ at $\left.C_{f 0}=0\right)$ is about $31^{\circ}$; it would be approached asymptotically as $R_{\Delta} \rightarrow \infty$. In practice, values of $C_{f 0}<0.0006$ can be obtained for adiabatic wall conditions in existing facilities at high (hypersonic) values of Mach number. However, the experimental results available for hypersonic turbulent boundary-layer separation are generally for a cold wall condition $\left(T_{w} / T_{r}<1\right)$ and so we have not included such data in Fig. 12.

\section{Discussion}

Possibly the most remarkable feature of the results presented above is the disappearance of the Mach-number dependence (for $M_{e} \geq 2.5$ ) of the interaction length and of the incipient-separation angle when they are plotted against the skin-friction coefficient. Although the correlation was found when looking for possible correlations with law-of-the-wall 
parameters, it is not possible to rationalize it in those terms. As previously noted, ${ }^{1,19,20}$ the interaction of a supersonic, turbulent boundary layer with a corner is controlled by a wall interaction layer that is considerably thicker than the sublayer and includes some supersonic portion. Increasing Reynolds number (decreasing $C_{f}$ ) "fills out" the velocity profile and brings the supersonic portion closer to the wall (relative to $\delta$ ), i.e., it reduces the wall-layer thickness and the related scale of the interaction. It is this effect that controls both the conditions for incipient separation and the interaction length, $\ell_{0}$. When the external Mach number is too low $\left(M_{e}<2\right)$, the Mach number at the edge of the interaction layer is low enough that the interaction becomes a "transonic" one with rather different characteristics from those at higher Mach numbers. We had noted this in the experiments of Ref. 1. For example, at $M_{e}=1.95$, the shock wave ahead of the corner was detached, even for values of $\alpha$ for which the boundary layer had not yet separated. That the present $M_{e}=1.98$ data do not fall on the correlation curves in Fig. 8 may be connected with such "transonic' behavior. In passing, we note that the $M_{e}=2$ data from Ref. 1 and from the present experiments consistently fall onto values of "effective $\alpha$ " about $4^{\circ}$ above those for the correlation curves in Fig. 8 .

To obtain a clearer understanding of these interactions requires a rational method for determining the thickness of the postulated wall interaction layer. Our own and other ${ }^{19,20}$ methods for defining it have been ad hoc. Explanations for the Mach-number independence in Fig. 8 and for the linear dependence on $C_{f}$ await a better understanding of the wall interaction layer.

An important point is that the present correlation is strictly for the adiabatic condition, $T_{w}=T_{r}$. It does not apply for $T_{w}$ $\neq T_{r}$, even if the effects of heat transfer on $C_{f}$ are taken into account. For example, the effect of wall cooling $\left(T_{w}<T_{r}\right)$ is to increase $C_{f}$; from the present correlation we might conclude that there is a corresponding increase in $\ell_{0} / \delta_{0}$ but, in fact, a decrease is observed. ${ }^{16}$ The explanation is that wall cooling makes the velocity profile fuller and reduces the thickness of the wall interaction layer. In the present experiments, we saw evidence of such wall-temperature effects in Schlieren photographs taken immediately after the tunnel started at the time the total-temperature transient peaked. 'However, the data reported here and used in the correlations were obtained under stable, nearly constant total-temperature conditions for which the wall temperature was within $5 \%$ of the calculated recovery temperature.

With respect to the results for values of $\alpha$ greater than $30^{\circ}$, it may be seen from Fig. 8 that Mach-number independence still exists for $\alpha=35^{\circ}$ and $40^{\circ}$, but the data do not fall onto straight-line correlations (as explained earlier, the straight line shown for $\alpha=35^{\circ}$ was obtained "after the fact"). It is also noted that these values of $\alpha$ are large enough that the angles through which the separated shear layers must turn at reattachment are approaching the maximum values possible ${ }^{21}$ and even the maximum values possible for turning without shock detachment. Under these conditions, the reattachment point may be expected to move rapidly downstream (with incrasing $\alpha$ ), and the separation point correspondingly upstream. If the reattachment point reached the shoulder the flow would be basically the same as that ahead of a step, and the separation point would have reached a distance 4.2 step heights upstream of the shoulder. "In none of the data reported here had this condition yet been reached. Also, for these larger values of $\alpha$ and correspondingly larger separated regions, effect of axial symmetry can be expected, i.e., the separation region should be smaller than in a corresponding two-dimensional flow. This observation is supported by the experience reported in Ref. 21 and the data of Settles et al.

In regard to the problem of "incipient separation", the ambiguities that exist among its definitions and measurements are distressing from a strictly scientific point of view. On the other hand, the ambiguities can be accounted for in most practical cases by adopting either the "large" or "small" separation region criterion, as conditions warrant. Nevertheless, the need still exists for further clarification of the definition and its corresponding dependence upon the parameters of the problem. The last word on this problem has not yet been written.

\section{References}

'Roshko, A. and Thomke, G. J., "Supersonic Turbulent Boundary-Layer Interaction with a Compression Corner at Very High Reynolds Number," Proceedings of the Symposium on Viscous Interaction Phenomena in Supersonic and Hypersonic Flow, USAF Aerospace Research Labs., Wright-Patterson AFB, Ohio, Univ. of Dayton Press, 1969, pp. 109-138.

${ }^{2}$ Kuehn, D. M., "Experimental Investigation of the Pressure Rise Required for the Incipient Separation of Turbulent Boundary Layers in Two-Dimensional Supersonic Flow," NASA, Memo 1-21-59A, 1959.

${ }^{3}$ Settles, G. S. and Bogdonoff, S. M., "Separation of a Supersonic Turbulent Boundary Layer at Moderate to High Reynolds Number," AIAA Paper 73-666, Palm Springs, Calif., 1973.

${ }^{4}$ Law, C. H., "Supersonic, Turbulent Boundary Layer Separation Measurements at Reynolds Numbers of $10^{7}$ to $10^{8}$," AIAA Journal, Vol. 12, June 1974, pp. 794-797.

${ }^{5}$ Lighthill, M. J., "On Boundary Layers and Upstream Influence. II Supersonic Flow Without Separation." Proceedings of the Royal Society, A217, 1953, pp. 478-492.

${ }^{6}$ Kessler, T. J. and Page, R. H., "'Supersonic Turbulent Boundary Layer Separation Ahead of a Wedge," 10th Mid-western Mechanics Conference, Developments in Mechanics, Vol. 4, Colorado State University, Ft. Collins, Colo., Aug. 1967, pp. 1011-1028.

${ }^{7}$ Hunter, L. G. and Reeves, B. L., "Results of a Strong Interaction, Wake-Like Model of Supersonic Separated and Reattaching Turbulent Flows,"' AIAA Journal, Vol. 9, April 1971, pp. 703-712.

${ }^{8}$ Roshko, A. and Thomke, G. J., "Observations of Turbulent Reattachment Behind an Axisymmetric Downstream-Facing Step in Supersonic Flow," A A A A Journal, Vol. 4, June 1966, pp. 975-980.

${ }^{9}$ Pate, S. R. and Schueler, C. J., "Effects of Radiated Aerodynamic Noise on Model Boundary-Layer Transition in Supersonic and Hypersonic Wind Tunnels," USAF Arnold Eng. and Develop. Center, Tullahoma, Tenn., TR-67-236, Mar. 1968.

${ }^{10}$ Hopkins, E. J., "Charts for Predicting Turbulent Skin-Friction From the Van Driest Method (II)," NASA, TN D-6945, Oct. 1972.

${ }^{11}$ Zukoski, E. E., "Turbulent Boundary-Layer Separation in Front of a Forward-Facing Step," AIAA Journal, Vol. 5, Oct. 1967, pp. 1746-1753.

${ }^{12}$ Werle, M. J., "A Critical Review of Analytical Methods for Estimating Control Forces Produced by Secondary Injection," U.S. Naval Ordnance Lab., White Oak, Md., NOL TR 68-5, Jan. 1968.

${ }^{13}$ Thomke, G. J. and Roshko, A., "Incipient Separation of a Turbulent Boundary Layer at High Reynolds Number in TwoDimensional Supersonic Flow over a Compression Corner," McDonnell Douglas Astro. Co., Santa Monica, Calif., DAC-59819, Jan. 1969.

${ }^{14}$ Law, C. H., private communication, USAF Aerospace Research Labs., Wright-Patterson AFB, Ohio, Aug. 1974.

${ }^{15}$ Settles, G. S., Bogdonoff, S. M., and Vas, I. E., "Incipient Separation of a Supersonic Turbulent Boundary Layer at Moderate to High Reynolds Numbers,"' AIAA Paper 75-7, Pasadena, Calif., 1975.

${ }^{16}$ Spaid, F. W. and Frishett, J. C., "Incipient Separation of a Supersonic, Turbulent Boundary Layer, Including Effects of Heat Transfer,"' AIAA Journal, Vol. 19, July 1972, pp. 915-922.

${ }^{17}$ Rose, W. C., Page, R. J., and Childs, M. E., "Incipient Separation Pressure Rise for a Mach 3.8 Turbulent Boundary Layer," AIA A Journal, Vol. 11, May 1973, pp. 761-763.

${ }^{18}$ Appels, C., "Incipient Separation of a Compressible Turbulent Boundary Layer," von Karman Institute for Fluid Dynamics, RhodeSaint Genese, Belgium, T.N. 99, April 1974.

${ }^{19}$ Rose, W. C., Murphy, J. D., and Watson, E. C., "Interaction of an Oblique Shock Wave with a Turbulent Boundary Layer," AIAA Journal, Vol. 6, Sept. 1968, pp. 1792-1793.

${ }^{20}$ Elfstrom, G. M., "Turbulent Hypersonic Flow at a WedgeCompression Corner," Journal of Fluid Mechanics, Vol. 53, Jan. 1972, pp. 113-127.

${ }^{21}$ Sirieix, M., Délery, J., and Mirande, J., "Recherches Exp' erimentales Fondamentales Sur Les Ecoulements Séparés et Applications,"' La Recherche Aerospatiale, No. 520, 1967. 